\title{
An Overview of LoRaWAN
}

\author{
Biswajit Paul \\ Department of Electrical and Electronic Engineering \\ Shahjalal University of Science and Technology \\ Kumargaon, Sylhet-3114 \\ Bangladesh
}

\begin{abstract}
LoRa is an IoT enabling technology which is particularly suitable for low data rate applications. The technology can achieve extended network coverage while operating in unlicensed ISM band and falls into the category of Low-Power-Wide-Area-Networks (LPWANs) technologies. Among the non-cellular based LPWAN enabling technologies, LoRa has got remarkable attention due to its fast adoption by industries. LoRa through wireless modulation enables the end-nodes to establish long distance communication while LoRaWAN refers to the communication protocol and system architecture. In this paper, an overview of LoRaWAN is presented.
\end{abstract}

Key-Words: - IoT, LPWAN, LoRaWAN, Network Architecture, Link Design Factors, Transceivers.

Received: August 20, 2020. Revised: November 30, 2020. Accepted: December 10, 2020. Published: December 28, 2020.

\section{Introduction}

LoRa taking its name from 'Long Range', is a patented wireless data communication technology. The technology is developed by Cycleo of Grenoble, France [1] and was acquired by Semtech in 2012. The key feature that differentiates LoRa from other available wireless WAN technologies is long range transmission with low power consumption. However, it supports low bit rate applications with less demand on mobility and reliability. This technology is developed to support a type of wireless telecommunication wide area network; commonly known as Low Power Wide Area Networks (LPWANs), which is targeting to achieve a $+20 \mathrm{~dB}$ gain over legacy cellular system [2].

Although some traditional solutions like Bluetooth, Wi-Fi, ZigBee, WLAN, Z wave, cellular networks, GSM, LTE can provide wireless connection of the IoT devices in a network, those solutions demand high cost, high energy consumption and high complexity. European Telecommunications Standard Institute (ETSI), Third Generation Partnership Project (3 GPPP), Institute of Electrical and Electronic Engineers (IEEE) and Internet Engineering Task Force (IETF) are working towards the open standards for LPWAN technologies. Unlike some of the other LPWAN technologies that use unlicensed spectrum band, LoRa uses 128AES encryption on multiple levels for all data from sensors to application server as a mean to data protection and privacy [3]. Moreover, there is no technical obstacle to operating a LoRaWAN private solution in a licensed band although the technology is evolving based on unlicensed spectrum band [3].

According to experts and researchers, billions of IoT devices will be deployed in near future. LoRa is considered a very promising technology which is ex- pected to be an integral part of the enormous IoT market with a very good industrial support base. LoRa is currently targeting some specific IoT application sectors where Sigfox, Telensa, Weightless, DASH7, LTE Advanced for Machine Type Communications (LTE MTC), NarrowBandIoT (NB-IOT), Random Phase Multiple Access (RPMA) are some of the competing technologies.

The LoRa Alliance, a non-profit association which consists of more than 500 member companies is committed to enable large scale deployment of LPWAN IoT through the development and promotion of the LoRaWAN open standard. LoRa Alliance believes that LoRaWAN is a technology that complements LTE variations with an intention to serve different application segments [3]. According to LoRa Alliance web site, network operators predict that $10-15 \%$ of the predicted volume of IoT devices will be connected with cellular technologies and there will be a significant dependence on other LPWAN technologies to fill up the deficit. Developing a strong ecosystem for LoRaWAN is the primary objective behind forming this alliance.

Cisco, IBM, Semtech, Actility, Alibaba Group are some of the major sponsoring companies along with many other member companies in LoRa alliance. Among the members, Actility, Google Cloud, Semtech have a worldwide geographical focus, Alibaba Group has a geographical focus on Asia/South Pacific, IBM has a geographical focus on Europe and Cisco has its focus on North America and South America. Alibaba Group is currently focusing on the vertical markets of smart cities, smart industries/industrial IoT, smart homes consumer with an emphasis on software, platform and services. Google Cloud is focusing on much broader vertical markets such as smart agriculture, smart buildings, smart cities, smart environment, smart industry, smart in- 
frastructure etc. with an emphasis on IoT platform, platform development, storage etc.

LoRa commonly refer to two distinct layers [4]: (i) a physical layer using the Chirp Spread Spectrum (CSS) radio modulation technique and (ii) a MAC layer protocol (LoRaWAN), although the LoRa communications system also implies a specific access network architecture. However, LoRa is known as a physical layer technology which utilizing a proprietary spread spectrum technique, modulates the signals in sub-GHz ISM band [2]. The CSS technique of spreading a narrowband input signal over a wider channel bandwidth provides a bidirectional communication. The chirp signals generated by the transmitters vary their frequency over time without changing their phase between adjacent symbols. The CSS technique ensures phase continuity between different chirp symbols in the preamble part of the physical layer packet which enables simpler and more accurate timing and frequency synchronization and eliminates the need for expensive components to generate a stable local clock in the LoRa node [5]. It is possible to decode a severely attenuated signal several dBs below the noise floor by a distant receiver as long as the change in frequency is slow enough to put higher energy per chirp symbol. In each transmission, the payload can range from 2-255 octets.

In order to enable communication among many end devices and gateways (GW), a medium access control mechanism is required which is provided by LoRaWAN. According to the LoRaWAN specifications, the MAC layer is basically an ALOHA protocol which is controlled by LoRaNetServer [5]. LoRa MAC has been designed with an intention to mimic the interface of the IEEE 802.15.4 MAC which in turn enable accommodation of some major protocols such as 6LoWPAN and Constrained Application Protocol (CoAP). LoRa modulation which is developed and commercialized by Semtech Corporation is proprietary while LoRaWAN is an open standard which is developed by LoRa Alliance.

\section{LoRa Network Architecture and Elements}

The topology for LoRa networks is 'star-of-stars' and three different types of devices are supported by a typical LoRa network which are (i) end device (ii) LoRa gateway and (iii) LoRa network server. In this architecture, end devices communicate with the GWs using LoRa modulation following LoRaWAN. Raw LoRaWAN frames from the end devices are forwarded by the GWs to a network server over a backhaul interface typically through Ethernet or 3G. Gateways thus can be seen as bidirectional relays performing protocol conversion. Network server decodes the pack- ets sent by the devices and generates the packets that should be sent back to the devices. IEEE 64-bit extended unique identifier (EUI) is employed by LoRaWAN to automatically associate IPv6 addresses with LoRa nodes. In order to ensure security LoRaWAN employs several layers of encryption using (i) a unique network key to secure the network layer, (ii) a unique application key to provide end-to-end security at the application layer and (iii) a device specific key to ensure secure joining of a node to the network.

End devices (e.g: sensors) are the network elements that communicate with gateways using LoRa. Each end device has to be personalized and activated in order to participate in a LoRaWAN network. End devices can be activated either via Over-The-Air Activation (OTAA) when an end device is deployed or reset, or via Activation By Personalization (ABP) in which the two steps of end-device personalization and activation are done as one step. In Over-The-AirActivation process end-devices need to follow a network join procedure before data exchange with network server can take place. End-devices need to be personalized before the join procedure with the required information which are: a globally unique enddevice identifier (DevEUI), the application identifier (AppEUI) and an AES-128 key (AppKey). Three different classes of end devices namely Class-A, Class-B and Class- $\mathrm{C}$ are supported by LoRaWAN to address the various applications requirements.

Class-A: The default functional mode of LoRa networks is defined by Class-A devices and all LoRa devices must support this mode of operation [5]. ClassA devices support bidirectional traffic whereas an uplink transmission can be scheduled by the end devices based on their own needs [4]. Transmission initiated by the end devices in a very asynchronous manner is always the case in a Class-A network. Each uplink transmission is followed by two short downlink windows to receive any command or returned data packet from the network server and to increase resilience against channel fluctuations. Downlink transmission on the other hand can take place after the occurrence of an uplink transmission and thus will require a waiting period. Class-A devices consumes the lowest amount of power while offering less flexibility on downlink transmissions [4]. The main target applications for Class-A networks are the monitoring applications where the data produced by the end devices have to be collected by a control station [5].

Class-B: Class-B devices support bidirectional traffic with scheduled receive slots [4]. At scheduled times, extra receive windows are opened by Class-B devices. A synchronized beacon from the gateway enables the network server to know when the end devices are listening. Unlike Class-A devices, Class- 


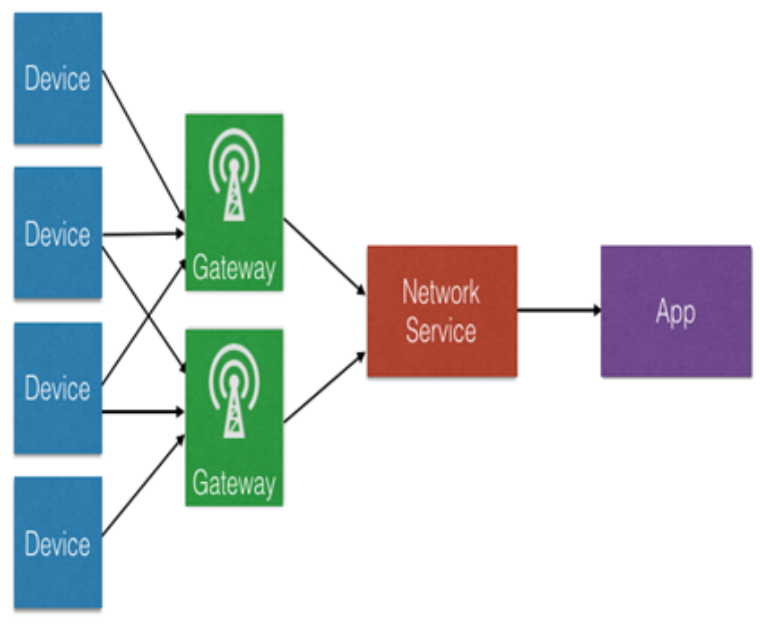

Figure 1: LoRa network topology

B end devices can receive downlink data or command packets from the network server irrespective of the uplink traffic and thereby decoupling uplink and downlink transmission [5]. Applications that require receiving commands from a remote controller are the main target applications for Class-B devices [5].

Class-C: Class-C devices support bidirectional traffic with maximal receive slots [4]. Class-C end devices while supporting almost continuous receive windows consume the maximum power among the three device classes. A possible application is power grid [5].

Gateway (GW) is the network element that works as a bridge between end devices and network servers. Packets generated from the end devices are forwarded to a network server by the GWs over an IP backhaul interface (e.g: Ethernet or 3G). More than one GW can be deployed in a LoRa network and a single packet can be received by multiple GWs.

Network server decodes the packets received from the end devices. De-duplicating of the packets is also performed by network server if the packets need to be sent back to the devices.

\section{LoRa Link Design Factors}

Spreading Factors: According to [6], In LoRa spread spectrum modulation is performed by representing each bit of payload information by multiple chips of information. Symbol rate (RS) refers to the rate at which the spread information is sent. Spreading factor (SF) is the ratio between the nominal symbol rate and chip rate and represents the number of symbols sent per bit of information. Spreading factors must be known in advance to both transmit and receive sides of a link as those spreading factors are orthogonal to each other [6]. However, there is a signal to noise (SNR) requirement associated with each of the spreading factors which need to be taken into account while deciding the link budget and range of LoRa receiver. Information about different spreading factors can be found in Table 1 [6]. SF6 is a special use case which provides the highest possible data rate transmission with LoRa modem. Typically SF7 to SF12 are used for uplink transmission.

Table 1: Chips/symbol rate and LoRa demodulator SNR requirement for different spreading factors

\begin{tabular}{|l|l|l|}
\hline $\begin{array}{l}\text { Spreading } \\
\text { Factor }\end{array}$ & $\begin{array}{l}\text { (Chips/ } \\
\text { symbol) }\end{array}$ & $\begin{array}{l}\text { LoRa De- } \\
\text { modulator } \\
\text { SNR (dB) }\end{array}$ \\
\hline 6 & 64 & -5 \\
\hline 7 & 128 & -7.5 \\
\hline 8 & 256 & -10 \\
\hline 9 & 512 & -12.5 \\
\hline 10 & 1024 & -15 \\
\hline 11 & 2048 & -17.5 \\
\hline 12 & 4096 & -20 \\
\hline
\end{tabular}

Coding Rate: LoRa modem employs cyclic error coding to perform forward error detection and correction and thereby introducing transmission overhead. Table 2 represents the transmission overhead associated with different cyclic coding rate [6]. Different coding rate options provide flexibility in responding different channel conditions. Through the employment of forward error correction LoRa networks can improve the reliability of a link in the presence of interference.

Table 2: Transmission overhead ratio associated with different coding rates

\begin{tabular}{|l|l|l|}
\hline $\begin{array}{l}\text { Coding } \\
\text { Rate }\end{array}$ & $\begin{array}{l}\text { Cyclic } \\
\text { Coding } \\
\text { Rate }\end{array}$ & $\begin{array}{l}\text { Overhead } \\
\text { Ratio }\end{array}$ \\
\hline 1 & $4 / 5$ & 1.25 \\
\hline 2 & $4 / 6$ & 1.5 \\
\hline 3 & $4 / 7$ & 1.75 \\
\hline 4 & $4 / 8$ & 2 \\
\hline
\end{tabular}

Signal Bandwidth: LoRaWAN supports the use of different bandwidths. A higher effective data rate can be achieved with a higher signal bandwidth which reduces transmission time at the expense of reduced sensitivity improvement. The LoRa modem bandwidth refers to the double sideband bandwidth. Variation in bit rate for different bandwidth options is highlighted in Table 3 [6]. However, it is important to note that permissible occupied bandwidth is governed by country specific regulatory constraints.

Adaptive Data Rate: Adaptive data rate (ADR) 
Table 3: Variation in bit rates for different bandwidth options

\begin{tabular}{|l|l|l|l|l|}
\hline $\begin{array}{l}\text { Bandwidth } \\
(\mathrm{KHz})\end{array}$ & $\begin{array}{l}\text { Spreading } \\
\text { Factor }\end{array}$ & $\begin{array}{l}\text { Coding } \\
\text { Rate }\end{array}$ & $\begin{array}{l}\text { Nominal } \\
R_{b}(\mathrm{bps})\end{array}$ & $\begin{array}{l}\text { Sensitivity } \\
(\mathrm{dBm})\end{array}$ \\
\hline 125 & 12 & $4 / 5$ & 293 & -136 \\
\hline 250 & 12 & $4 / 5$ & 586 & -133 \\
\hline 500 & 12 & $4 / 5$ & 1172 & -130 \\
\hline
\end{tabular}

scheme helps the end-devices to maximize both battery life and overall network capacity. End-devices can transmit on any channel depending on availability using any available data rate. However, the following rules must be followed:

i. For every transmission, the end-devices have to change channels in a pseudo-random fashion.

ii. End-devices should not violate the maximum transmit duty cycle or maximum transmit duration (dwell time) relative to the sub-band used and local regulations.

Packet Structure: LoRa packet consist of three elements: a preamble, an optional header and the data payload [6].

Preamble: The preamble is used for the purpose of synchronization between the receiver and the incoming data flow. It is a programmable variable which means the preamble length may be extended as required by applications whereas the default configuration is a 12 symbol long sequence for the packet. A preamble detection process is undertaken by the receiver that periodically restarts and therefore the preamble length should be configured identical to the transmitter preamble length. The maximum preamble length should be programmed on the receiver side if the preamble length is not known or can vary.

Header: Two types of header are available based on the chosen mode of operation which are: explicit header mode and implicit header mode. Explicit header mode is the default mode of operation where the header provides information about the payload length in bytes, the forward error correction code rate and the presence of an optional 16-bits CRC for the payload. In cases where the payload, coding rate and CRC presence are fixed or known in advance, implicit header mode might be chosen over explicit header mode to reduce transmission time.

Payload: The packet payload is a variable-length field which contains the actual data coded at the error rate. The error rate is specified in the header in explicit mode or in the register settings in implicit mode.

Figure 2 shows the packet structure used in LoRaWAN [7] and Table 4 represents the meaning of the abbreviations used in those figures.

The minimum payload length $\left(L_{D P}\right)$ can be ex-

\begin{tabular}{|c|c|c|c|c|}
\hline Preamble & PHDR & PHDR_CRC & PHYPayload & CRC \\
\hline \multicolumn{5}{|c|}{ Uplink PHY Structure } \\
\hline Preamble & PHDR & PHDR_CRC & PHYPayload \\
\hline \multicolumn{4}{|c|}{ Dowullink PHY Structure } \\
\hline MHDR & \multicolumn{2}{|c|}{ MACPayload } & MIC \\
\hline \multicolumn{4}{|c|}{ PHY Payload Structure } \\
\hline FHDR & \multicolumn{2}{|c|}{ FPort } & FRMPayload \\
\hline \multicolumn{4}{|c|}{ MAC Payload Structure } \\
\hline DevAddr & FCril & FCHt & FOpts \\
\hline
\end{tabular}

Figure 2: Packet structure in LoRaWAN

Table 4: Abbreviation and meaning used in packet structure

\begin{tabular}{|l|l|}
\hline Abbreviation & Meaning \\
\hline PHDR & Physical header \\
\hline MHDR & MAC header \\
\hline MIC & Message integrity code \\
\hline FHDR & Frame header \\
\hline FPort & Application specific port \\
\hline DevAddr & $\begin{array}{l}\text { Length of ED address } \\
\text { field }\end{array}$ \\
\hline FCtrl & $\begin{array}{l}\text { Length of the FHDR's } \\
\text { frame control }\end{array}$ \\
\hline FCnt & Frame counter fields \\
\hline Fopts & $\begin{array}{l}\text { Length of the optional } \\
\text { FHDR field carrying } \\
\text { MAC commands }\end{array}$ \\
\hline
\end{tabular}

pressed as [7],

$$
\begin{aligned}
& L_{D P}=\text { MHDR }+ \text { MACPayload }+ \text { MIC } \\
& =\text { MHDR }+ \text { DevAddr }+ \text { FCtrl }+ \text { FCnt }+ \\
& \text { Fopts }+ \text { Fport }+ \text { FRMPayload }+ \text { MIC } \\
& =1 \text { byte }+ \text { 4bytes }+1 \text { byte }+2 \text { bytes }+1 \text { byte }+ \\
& \text { Fport }+ \text { FRMPayload }+ \text { 4bytes } \\
& =13 \text { bytes }+ \text { Fopts }+ \text { FRMPayload }
\end{aligned}
$$




\subsection{Important Expressions Related to Transmissions}

Symbol rate $\left(R_{s}\right)$ : In LoRaWAN, the symbol rate $\left(R_{s}\right)$ can be expressed in terms of channel bandwidth $(B W)$ and spreading factor $(S F)$ in the following manner [6],

$$
R_{s}=\frac{B W}{2^{S F}}
$$

Data rate $\left(R_{b}\right)$ : Data bit rate $\left(R_{b}\right)$ can be calculates as [48],

$$
R_{b}=S F \times \frac{\frac{4}{4+C R}}{\frac{2^{S F}}{B W}}
$$

The symbol period $\left(T_{s}\right)$ can be calculated as,

$$
T_{s}=\frac{1}{R_{s}}
$$

Time on Air ( $T o A)$ calculation: ToA refers to the interval between the first bit of a message frame leaving the ED and the last bit of that message frame leaving the ED. ToA can be written as,

$$
T o A=T_{\text {preamble }}+T_{\text {payload }},
$$

Where $T_{\text {preamble }}$ and $T_{\text {payload }}$ are given by,

$$
T_{\text {preamble }}=\left(n_{\text {preamble }}+4.25\right) \times T_{s},
$$

and

$$
T_{\text {payload }}=n_{\text {paylaod }} \times T_{s},
$$

Where $n_{\text {preamble }}$ and $n_{\text {payload }}$ are the length of the preamble and payload respectively. $n_{\text {payload }}$ can be found as,

$$
\begin{gathered}
n_{\text {payload }}=(S W+ \\
\max \left(\left(\operatorname{ceil}\left(\frac{8 P L-4 S F+28+16 C R C-20 I H}{4(S F-2 D E)}\right)\right.\right. \\
(C R+4)), 0)))
\end{gathered}
$$

For LoRA channel,

$$
\begin{gathered}
\text { ToA }=\frac{2^{S F}}{B W}\left(\left(n_{\text {premble }}+4.25\right)+(S W+\right. \\
\max \left(\left(\operatorname{ceil}\left(\frac{8 P L-4 S F+28+16 C R C-20 I H}{4(S F-2 D E)}\right)\right.\right. \\
(C R+4)), 0)))
\end{gathered}
$$

For GFSK channel,

$$
T o A=\frac{8}{D R}\left(n_{\text {preamble }}+S W+P L+2 C R C\right)
$$

Where, $n_{\text {preamble }}=8$ bytes for LoRa channel and $n_{\text {preamble }}=5$ bytes for GFSK channel. $S W$ is the length of the synchronization word. $S W=8$ bits for LoRa channel and $S W=3$ bytes for GFSK channel. The presence of the payload is indictaed by $C R C$. $C R C=1$ when on and $C R C=0$ when off. $I H=1$ when implicit header mode is enabled and $I H=0$ when explicit header mode is enabled. $D E=1$ for low data rate optimization with $S F=11 / 12$ and $B W=125 \mathrm{KHz}$ and $D E=0$ for all other cases. $\mathrm{CR}$ represents the coding rate and the value can range from 1 to $4 . D R=50 \mathrm{kbits} / \mathrm{s}$ for GFSK channel.

\section{Geographical Bands}

LoRa Alliance has drafted out country specific band of operation and channel plan [9]. Operation guidelines for EU863-870 MHz ISM band are discussed in this section.

EU863-870 MHz ISM Band: ISM radio spectrum allocation in Europe is defined by ETSI [EN300.220]. While the network channels can be freely attributed by the network operator, three default channels must be implemented in every end-device. Those channels are $868.10 \mathrm{MHz}, 868.30 \mathrm{MHz}$ and $868.50 \mathrm{MHz}$ with LoRa modulation, $125 \mathrm{KHz}$ bandwidth occupancy. Those channels form the minimum set that all network gateways should always be listening on. A maximum of 16 channels can be supported by EU863-870 LoRaWAN. Different data rates and transmit power configurations are supported as shown in Table 5 [9]. End-devices feature a channel data structure to store the parameters of at least 16 channels whereas a channel structure corresponds to a frequency and a set of data rates usable on this frequency. The default radiated transmit output power is $14 \mathrm{dBm}$ for EU868 $\mathrm{MHz}$ ISM band end-devices. The maximum MACPayload size length (M) and the maximum application payload length in the absence of the optional FOpt control field $(\mathrm{N})$ supported by the end-devices operating in EU868 MHz band are given in Table 6 [9].

ETSI regulations [10] impose some restrictions in order to access the physical medium. The maximum time a transmitter can be on or the maximum time a transmitter can transmit per hour is governed by ETSI regulations. The ETSI regulations allow to choose one of the two choices which are duty-cycle limitation or a so called Listen Before Talk Adaptive Frequency Agility (LBT AFA) transmissions management. However, in order to comply with the ETSI regulations, the current LoRaWAN specification exclusively uses duty-cycled limited transmissions [9]. Re- 
Table 5: Different data rates and transmit power supported by EU868 MHz ISM Band

\begin{tabular}{|c|c|c|c|}
\hline $\begin{array}{l}\text { Data } \\
\text { Rate }\end{array}$ & Configuration & $\begin{array}{l}\text { Bit Rate } \\
\text { (bits/s) }\end{array}$ & $\begin{array}{l}\text { Transmit } \\
\text { Power } \\
(\mathrm{dBm})\end{array}$ \\
\hline 0 & $\begin{array}{l}\text { LoRa: } \\
\text { SF12/125 } \\
\text { KHz }\end{array}$ & 250 & 20 \\
\hline 1 & $\begin{array}{l}\text { LoRa: } \\
\text { SF11/125 } \\
\mathrm{KHz}\end{array}$ & 440 & 14 \\
\hline 2 & $\begin{array}{l}\text { LoRa: } \\
\text { SF10/125 } \\
\text { KHz }\end{array}$ & 980 & 11 \\
\hline 3 & $\begin{array}{l}\text { LoRa: SF9/125 } \\
\text { KHz }\end{array}$ & 1760 & 8 \\
\hline 4 & $\begin{array}{l}\text { LoRa: SF8/125 } \\
\mathrm{KHz}\end{array}$ & 3125 & 5 \\
\hline 5 & $\begin{array}{l}\text { LoRa: SF7/125 } \\
\text { KHz }\end{array}$ & 5470 & 2 \\
\hline 6 & $\begin{array}{l}\text { LoRa: SF7/250 } \\
\text { KHz }\end{array}$ & 11000 & RFU \\
\hline 7 & FSK: $50 \mathrm{Kbps}$ & 50000 & RFU \\
\hline $8-15$ & RFU & RFU & RFU \\
\hline
\end{tabular}

strictions on duty cycle and effective radiated power are provided in Table 7 [10].

Table 6: Maximum payload size supported by enddevices operating in EU868 MHz ISM band

\begin{tabular}{|l|l|l|}
\hline Data Rate & M & N \\
\hline 0 & 59 & 51 \\
\hline 1 & 59 & 51 \\
\hline 2 & 59 & 51 \\
\hline 3 & 123 & 115 \\
\hline 4 & 230 & 222 \\
\hline 5 & 230 & 222 \\
\hline 6 & 230 & 222 \\
\hline 7 & 230 & 222 \\
\hline $8: 15$ & Not defined & Not defined \\
\hline
\end{tabular}

A per sub-band duty-cycle limitation is enforced by the LoRaWAN [9]. When a frame is transmitted in a given sub-band, the time of emission and the on-air duration of the frame are recorded for that sub-band and the same sub-band cannot be used again for next Tof $f_{\text {subband }}$ which can be calculated using expression (11) [9]. The device can still transmit on a different sub-band during the unavailable time of a given sub-band. The devices have to wait before transmis-
Table 7: Duty cycle and transmit power restriction on end-devices operating in EU868 MHz ISM band

\begin{tabular}{|l|l|l|}
\hline $\begin{array}{l}\text { Frequency Band } \\
\text { (MHz) }\end{array}$ & $\begin{array}{l}\text { Duty Cy- } \\
\text { cle (\%) }\end{array}$ & $\begin{array}{l}\text { Maximum } \\
\text { Tx Power } \\
\text { (mW, ERP) }\end{array}$ \\
\hline $\begin{array}{l}863-868.6,868.7- \\
869.2, \quad 869.4-\end{array}$ & 0.1 & 25 \\
$869.65,869.7-870$ & & \\
\hline $868.0-868.6$ & 1 & 25 \\
\hline $868.7-869.2$ & 0.1 & 25 \\
\hline $869.4-869.65$ & 10 & 500 \\
\hline $869.7-870.0$ & 1 & 25 \\
\hline $870.0-873.0$ & 1 & 25 \\
\hline
\end{tabular}

sion if all sub-bands are unavailable.

$$
\text { Toff } f_{\text {subband }}=\frac{\text { ToA }}{\text { DutyCycle } \text { subband }}-T o A(11)
$$

The first receive window RX1 uses the same channel as the preceding uplink and the data rate is a function of the uplink data rate while the allowed values for RX1DROffset are in the [0:5] range as shown in Table 8 [9]. For future use values in [6:7] are reserved. The second receive window RX2 uses a fixed frequency and data rate and the default parameters are 869.525 MHz/DR0 (SF12, $125 \mathrm{KHz}$ ).

\section{Transceivers}

Transceivers are important to determine link budget requirements. There are different commercially available transceivers in the market at present which can be used in LoRa networks such as SX1272, SX1276, SX1278. Information about SX1272, SX1276 and SX1278 can be found in [6] and [12] respectively. However, SX1272 transceiver is used for discussion here.

A maximum link budget of $157 \mathrm{~dB}$ can be achieved using SX1272 transceiver. Different modulations are supported such as FSK, GFSK, MSK, GMSK, LoRa and OOK. $10 \mathrm{~mA}$ RX current is reported in [6]. Intended application scenarios include automated meter reading, home and building automation, wireless alarm and security systems, industrial monitoring and control and long range irrigation system. Power consumption specification for SX1272 transceiver is provided in Table 9 [6]. For LoRa modulation $1 \%$ packet error rate (PER) is mentioned in the electrical specification. There are slight variations in the receive currents for different bandwidth options. Receiver sensitivities associated with different bandwidth options and different spreading factors are provided in Table 10 [6]. 
Table 8: Downlink RX1 data rate mapping

\begin{tabular}{|l|l|l|l|l|l|l|}
\hline $\begin{array}{l}\text { RX1DROffset } \\
\text { Upstream } \\
\text { Data Rate }\end{array}$ & 0 & 1 & 2 & 3 & 4 & 5 \\
\hline DR0 & DR0 & DR0 & DR0 & DR0 & DR0 & DR0 \\
\hline DR1 & DR1 & DR0 & DR0 & DR0 & DR0 & DR0 \\
\hline DR2 & DR2 & DR1 & DR0 & DR0 & DR0 & DR0 \\
\hline DR3 & DR3 & DR2 & DR1 & DR0 & DR0 & DR0 \\
\hline DR4 & DR4 & DR3 & DR2 & DR1 & DR0 & DR0 \\
\hline DR5 & DR5 & DR4 & DR3 & DR2 & DR1 & DR0 \\
\hline DR6 & DR6 & DR5 & DR4 & DR3 & DR2 & DR1 \\
\hline DR7 & DR7 & DR6 & DR5 & DR4 & DR3 & DR2 \\
\hline
\end{tabular}

Table 9: Power consumption specification for SX1272 transceiver

\begin{tabular}{|c|c|c|c|c|}
\hline Description & Conditions & Typical & Maximum & Unit \\
\hline $\begin{array}{l}\text { Supply current in Sleep } \\
\text { mode }\end{array}$ & - & 0.1 & 1 & $\mu \mathrm{A}$ \\
\hline $\begin{array}{l}\text { Supply current in Idle } \\
\text { mode }\end{array}$ & $\begin{array}{l}\mathrm{RC} \text { oscillator } \\
\text { enabled }\end{array}$ & 1.5 & - & $\mu \mathrm{A}$ \\
\hline $\begin{array}{l}\text { Supply current in standby } \\
\text { mode }\end{array}$ & $\begin{array}{l}\text { Crystal oscilla- } \\
\text { tor enabled }\end{array}$ & 1.4 & 1.6 & $\mathrm{~mA}$ \\
\hline $\begin{array}{l}\text { Supply current in synthe- } \\
\text { sizer mode }\end{array}$ & FSRx & 4.5 & - & $\mathrm{mA}$ \\
\hline $\begin{array}{l}\text { Supply current in receive } \\
\text { mode }\end{array}$ & LnaBoost off & 10.5 & - & $\mathrm{mA}$ \\
\hline $\begin{array}{l}\text { Supply current in receive } \\
\text { mode }\end{array}$ & LnaBoost on & 11.2 & - & $\mathrm{mA}$ \\
\hline $\begin{array}{l}\text { Supply current in trans- } \\
\text { mit mode with impedance } \\
\text { matching }\end{array}$ & $\begin{array}{l}\text { RFOP }=+20 \\
\mathrm{dBm} \text { on PA- } \\
\text { BOOST }\end{array}$ & 125 & - & $\mathrm{mA}$ \\
\hline $\begin{array}{l}\text { Supply current in trans- } \\
\text { mit mode with impedance } \\
\text { matching }\end{array}$ & $\begin{array}{l}\text { RFOP }=+17 \\
\mathrm{dBm} \text { on PA- } \\
\text { BOOST }\end{array}$ & 90 & - & $\mathrm{mA}$ \\
\hline $\begin{array}{l}\text { Supply current in trans- } \\
\text { mit mode with impedance } \\
\text { matching }\end{array}$ & $\begin{array}{l}\text { RFOP }=+13 \\
d B m \text { on } R F O \\
\text { pin }\end{array}$ & 28 & - & $\mathrm{mA}$ \\
\hline $\begin{array}{l}\text { Supply current in trans- } \\
\text { mit mode with impedance } \\
\text { matching }\end{array}$ & $\begin{array}{l}\text { RFOP }=+7 \mathrm{dBm} \\
\text { on RFO pin }\end{array}$ & 18 & - & $\mathrm{mA}$ \\
\hline
\end{tabular}

It can be seen from Table 9 that maximum energy is consumed by the end-nodes for packet transmission and reception. Transmit current increases along with increasing transmit power and thus, nodes located far away from the GW will consume more power to communicate with the GW. Receiver sensitivity decreases as the spreading factor increases as suggested by Table 10. While higher spreading factors support higher data rates, the choice of higher spreading factor decreases the maximum attainable link budget. Also, selection of higher bandwidths decreases the maximum attainable link budget as well which limits maximum achievable transmit distance.

\section{Research Directions}

Different aspects of LoRa technology have got the attention of researchers. A LoRa gateway is expected to serve thousands of nodes and since node to GW communication in LoRaWAN is similar to ALOHA scheme, interference can severely limit network performance. As a result, packet error ratio can be quite high in a congested network. Some works relevant to LoRa network interference and/or packet error ratio can be found in [13-15]. Some authors also believe 
Table 10: Receiver sensitivities associated with different bandwidth options and spreading factors

\begin{tabular}{|l|l|l|l|}
\hline Description & Conditions & Typical & Unit \\
\hline RF sensitivity, & $\mathrm{SF}=6$ & -121 & $\mathrm{dBm}$ \\
Long-Range Mode, & $\mathrm{SF}=7$ & -124 & $\mathrm{dBm}$ \\
highest LNA gain, & $\mathrm{SF}=8$ & -127 & $\mathrm{dBm}$ \\
LNA boost, & $\mathrm{SF}=9$ & -130 & $\mathrm{dBm}$ \\
125 KHz & $\mathrm{SF}=10$ & -133 & $\mathrm{dBm}$ \\
bandwidth using & $\mathrm{SF}=11$ & -135 & $\mathrm{dBm}$ \\
split Rx/Tx path & $\mathrm{SF}=12$ & -137 & $\mathrm{dBm}$ \\
\hline RF sensitivity, & $\mathrm{SF}=6$ & -118 & $\mathrm{dBm}$ \\
Long-Range Mode, & $\mathrm{SF}=7$ & -122 & $\mathrm{dBm}$ \\
highest LNA gain, & $\mathrm{SF}=8$ & -125 & $\mathrm{dBm}$ \\
LNA boost, & $\mathrm{SF}=9$ & -128 & $\mathrm{dBm}$ \\
250 KHz & $\mathrm{SF}=10$ & -130 & $\mathrm{dBm}$ \\
bandwidth using & $\mathrm{SF}=11$ & -132 & $\mathrm{dBm}$ \\
split Rx/Tx path & $\mathrm{SF}=12$ & -135 & $\mathrm{dBm}$ \\
\hline RF sensitivity, & $\mathrm{SF}=6$ & -111 & $\mathrm{dBm}$ \\
Long-Range Mode, & $\mathrm{SF}=7$ & -116 & $\mathrm{dBm}$ \\
highest LNA gain, & $\mathrm{SF}=8$ & -119 & $\mathrm{dBm}$ \\
LNA boost, & $\mathrm{SF}=9$ & -122 & $\mathrm{dBm}$ \\
500 KHz & $\mathrm{SF}=10$ & -125 & $\mathrm{dBm}$ \\
bandwidth using & $\mathrm{SF}=11$ & -128 & $\mathrm{dBm}$ \\
split Rx/Tx path & $\mathrm{SF}=12$ & -129 & $\mathrm{dBm}$ \\
\hline
\end{tabular}

that through some changes in the LoRaWAN protocol, LoRa networks can be benefited from different aspects. Authors in [16] proposed an adaptive duty cycle medium access control protocol to make networks more energy efficient which can also help to avoid excess congestion. Authors in [17] proposed a lightweight scheduling scheme to improve reliability and scalability of LoRa networks. The scalability issue of LoRa networks are explored in [18-19]. Battery lifetime for different classes of devices is explored in [8]. Prospects of multi-hop routing for LoRa networks can be found in [20] and impacts of packet re-transmission in LoRa networks can be found in [21].

Some potential applications of LoRa have been investigated as well in [1, 22-27]. A sailing monitoring system based on LoRa was studied in [22]. Authors in [1] proposed river water pollution monitoring using LoRa technology. Authors in [23] manufactured a prototype through integration of temperature and relative humidity sensor DHT22 with RF module for the purpose of sensing the physical world in harsh environment. A wireless paging system network was proposed for an elderly care system in [24]. SX1278 LoRa transceiver was used for radio transmission. Suitability of LoRa networks for the implementation of distributed management system in the field of smart metering, smart building monitoring and process industry was investigated in [25]. the au- thors in [26] proposed multi-hop transmission to monitor spatio-temporal dynamics of rainfall runoff and in-sewer processes and to monitor water resources in urban settlement. In [27], the authors made an interesting proposal to use LoRa protocol for the IoT deployment network and NB-IoT for gateway to cloud server communication

\section{Conclusion}

LoRa technology has the potential to be utilized in various emerging IoT applications. However, it is very important to know the technological features to determine its fitting use under different circumstances. Nevertheless, Network designers will have to carefully go through the details of LoRa technology like any other technology to extract the maximum benefits in terms of key network performance indicators. In this paper, network architecture, different elements, important inter-relationships among different variables, current communication protocol, regional restrictions and role of transceivers in network design are discussed.

\section{Acknowledgement}

This project was supported in part by University of Saskatchewan and in part by University Research Center, Shahjalal University of Science and Technology under the research grant: AS/2020/1/31. The author would like to thank Professor Ha Nguyen and Professor Francis Bui for their valuable suggestions.

\section{References:}

[1] P. Kumari, H. P. Gupta \& T. Dutta, A Stackelberg Game based River Water Pollution Monitoring System using LoRa Technology. In 2019 16th Annual IEEE International Conference on Sensing, Communication, and Networking (SECON) pp. 1-5, June, 2019.

[2] U. Raza, P. Kulkarni \& M. Sooriyabandara, Low power wide area networks: An overview, IEEE Communications Surveys \& Tutorials, Vol.19, No.2, 2017, pp. 855-873.

[3] https://lora-alliance.org/

[4] A. Augustin, J. Yi, T. Clausen \& W. M. Townsley, A study of LoRa: Long range low power networks for the internet of things, Sensors, Vol.16, No.9, 2016, pp. 1466.

[5] M. Centenaro, L. Vangelista, A. Zanella \& M. Zorzi, Long-range communications in unlicensed bands: The rising stars in the IoT and smart city scenarios, IEEE Wireless Communications, Vol.23, No.5, 2016, pp. 60-67. 
[6] "SX1272/73 - $860 \mathrm{MHz}$ to $1020 \mathrm{MHz}$ Low Power Long Range Transceiver, 2015. Datasheet."

[7] L. Alliance, Lorawan specification. LoRa Alliance, 2015.

[8] P. J. San Cheong, Bergs, C. Hawinkel \& J. Famaey, Comparison of LoRaWAN classes and their power consumption. 2017 IEEE Symposium in Communications and Vehicular Technology (SCVT), pp. 1-6, November, 2017.

[9] L. Alliance, LoRaWANTM 1.1 Regional Parameters, LoRa Alliance, 2017.

[10] "Regulation on collective frequencies for licence-exempt radio transmitters and on their use," Finnish Communications Regulatory Authority, Helsinki, Finland, FICORA 15 AI/2015 M, Dec. 2015.

[11] Wireless and Sensing Products, Semtech, FCC Regulations for ISM Band Devices: 902 - 928 $\mathrm{MHz}, 2006$.

[12] “SX1276/77/78 - 137-1050 MHz Ultra Low Power Long Range Transceiver, Datasheet, 2012".

[13] D. Bankov, E. Khorov \& A. Lyakhov, Mathematical model of LoRaWAN channel access, In Proceedings of the IEEE 18th International Symposium on A World of Wireless, Mobile and Multimedia Networks (WoWMoM), pp. 60-67, Macao, China, 2017.

[14] V. Gupta, S. K. Devar, N.H. Kumar \& K. P. Bagadi, Modelling of IoT Traffic and Its Impact on LoRaWAN, In GLOBECOM 20172017 IEEE Global Communications Conference, Vol.23, No.5, 2016, pp. 1-6, December, 2017.

[15] Z. Li, S. Zozor, J. M. Drossier, N. Varsier \& Q. Lampin, 2D Time-frequency interference modelling using stochastic geometry for performance evaluation in Low-Power Wide-Area Networks, In 2017 IEEE International Conference on Communications (ICC), pp. 1-7, May, 2017.

[16] T. Deng, J. Zhu \& Z. Nie, An improved LoRaWAN protocol based on adaptive duty cycle, In 2017 IEEE 3rd Information Technology and Mechatronics Engineering Conference (ITOEC), pp. 1122-1125, October, 2017.

[17] B. Reynders, Q. Wang, P. Tuset-Peiro, X. Vilajosana \& S. Pollin, Improving Reliability and Scalability of LoRaWANs Through Lightweight
Scheduling, IEEE Internet of Things Journal, Vol.5, No.3, 2018, pp. 1830-1842.

[18] K. Mikhaylov, J. Petäjäjärvi \& J. Janhunen, On LoRaWAN scalability: Empirical evaluation of susceptibility to inter-network interference, In 2017 European Conference on Networks and Communications (EuCNC), pp. 1-6, June, 2017.

[19] O. Georgiou \& U. Raza, Low power wide area network analysis: Can LoRa scale?, IEEE Wireless Communications Letters, Vol.6, No.2, 2017, pp. 162-165.

[20] B. Paul, A novel energy-efficient routing scheme for LoRa networks, IEEE Sensors Journal, Vol.20, No.15, 2020, pp. 1-4.

[21] B. Paul, A Novel Mathematical Model to Evaluate the Impact of Packet Retransmissions in LoRaWAN, IEEE Sensors Letters, Vol.4, No.5, 2020, pp. 8858-8866.

[22] L. Li, J. Ren, \& Q. Zhu, On the application of LoRa LPWAN technology in Sailing Monitoring System, In 2017 13th Annual Conference on Ondemand Network Systems and Services (WONS), pp.77-80, February, 2017.

[23] G. Loubet, A. Takacs \& D. Dragomirescu, Implementation of a battery-free wireless sensor for cyber-physical systems dedicated to structural health monitoring applications, IEEE access, vol. 7, 2019, pp. 24679-24690.

[24] G. Yang \& H. Liang, A smart wireless paging sensor network for elderly care application using LoRaWAN, IEEE Sensors Journal, vol.18, no. 22, 2018, pp. 9441-9448.

[25] M. Rizzi, P. Ferrari, A. Flammini \& E. Sisinni, Evaluation of the IoT LoRaWAN solution for distributed measurement applications, IEEE Transactions on Instrumentation and Measurement, vol. 66, no. 12, 2017, pp. 3340-3349.

[26] C. Ebi, F. Schaltegger, A. Rust \& F. Blumensaat, Synchronous LoRa "mesh network to monitor processes in underground infrastructure, IEEE Access, vol. 7, 2019, pp. 57663-57677.

[27] S. Gao, G. Y. Tian, X. Dai, M. Fan, X. Shi,J. Zhu \& K. Li, A Novel Distributed Linear-SpatialArray Sensing System Based on Multichannel LPWAN for Large-Scale Blast Wave Monitoring, IEEE Internet of Things Journal, vol. 6, no. 6, 2019, pp. 9679-9688.

\section{Creative Commons Attribution License 4.0 (Attribution 4.0 International, CC BY 4.0)}

This article is published under the terms of the Creative Commons Attribution License 4.0

https://creativecommons.org/licenses/by/4.0/deed.en_US 